\title{
A Hybrid Method for Real-Time Bronchoscope Tracking Using Contour Registration and Synchronous EMT Data
}

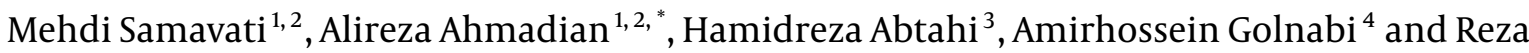 \\ Arjmandi Asl ${ }^{2}$ \\ ${ }^{1}$ Department of Medical Physics and Biomedical Engineering, School of Medicine, Tehran University of Medical Sciences, Tehran, Iran \\ ${ }^{2}$ Research Center for Biomedical Technologies and Robotics (RCBTR), Tehran University of Medical Sciences, Tehran, Iran \\ ${ }^{3}$ Department of Internal Medicine, School of Medicine, Tehran University of Medical Sciences, Tehran, Iran \\ ${ }^{4}$ Department of Mathematical Sciences, Montclair State University, New Jersey, United States \\ "Corresponding author: Department of Medical Physics and Biomedical Engineering, School of Medicine, Research Center for Biomedical Technologies and Robotics (RCBTR), \\ Tehran University of Medical Sciences, Tehran, Iran. Email: ahmadian@tums.ac.ir
}

Received 2018 February 01; Revised 2019 January 28; Accepted 2019 January 30.

\section{Abstract}

Background: Bronchoscopy is a difficult procedure for physicians to relate CT slices to bronchoscopic video images and maneuver the bronchoscope inside the airway tree. CT-guided bronchoscopy systems have been developed in the last decades to help physicians maneuver the bronchoscope inside the airway tree in a fast and precise way.

objectives: We aimed to develop a continuous guiding method for bronchoscopy with high tracking accuracy by matching bronchoscopy image contours with CT contours, and speed it up by using synchronous electromagnetic tracker (EMT) data, and to evaluate it on airway phantom with simulated respiratory motion.

Materials and Methods: This method works based on two approaches combined together: 1) Contours detected in real bronchoscopy images and finding their equivalents in CT space. Contours are detected by a fast algorithm and CT contours are mapped by them in a perspective scheme. 2) EMT data which is used in a frame by frame approach to approximate the position of bronchoscope compared to its previous position. This differential approach causes a small search space and as a result higher tracking speed. The novelty of this work is using bronchoscopy image contours instead of the whole image combined with synchronous EMT data. This approach causes faster tracking and there is no need for landmark selection or centerline consideration before performing the main bronchoscopy.

Results: The experimental results of implementing the proposed method show that this method can track the bronchoscope continuously. For evaluating the accuracy and robustness of tracking, virtual bronchoscopy images were generated at each frame position reported by the method and compared to corresponding real bronchoscopy image using mutual information. The experimental results present that this method can track a bronchoscope accurately and robustly in $96.3 \%$ of frames.

Conclusion: Using contours instead of the whole image for registration can provide a continuous real-time bronchoscopy tracking procedure. Using EMT data in differential mode makes the proposed method robust to simulated respiratory motion.

Keywords: CT-Guided Bronchoscopy, Bronchoscopy Navigation, Contour-Based 2D/3D Image Registration, Hybrid Tracking

\section{Background}

Bronchoscopy is a minimally invasive method to investigate inside the airway tree structure, for lung cancer diagnosis and staging. Before bronchoscopy operation, pulmonary nodules are detected on computed tomography (CT) scans of patients. Afterward, a bronchoscope is maneuvered through the airways to a region near the nodule. Transbronchial biopsy (TBB) is then performed to biopsy nodules.

This procedure has some difficulties for physicians. The physician should relate CT slices to bronchoscopic video images mentally and has no guidance to maneuver the bronchoscope through true branches to reach the target.

Image guided bronchoscopy systems have been developed in the last decades to help physicians navigate bronchoscope to reach target in a precise and fast way. These systems might track and show the bronchoscope's tip position on CT-driven airway tree structure.

Bronchoscopy tracking methods are categorized to image-based (1-5), electromagnetic tracker (EMT) based (6$9)$, and hybrid methods $(10,11)$.

Image-based methods usually use CT-derived virtual 
bronchoscopy (VB). Comparing similarity between real bronchoscopy video frames and VB images at different positions of virtual camera, the position of the tip of the bronchoscope in the CT coordinate system is acquired. Although these methods can reach the position precisely, they cannot track bronchoscope in real time. This problem is caused by time consumed by VB generation at different positions for each live video frame and measuring their similarity with each live video frame that arrives.

EMT systems have an electromagnetic field generator that induces voltage in tiny coils embedded in a sensor. They can report the sensor's position with high frame rate, however they suffer intrinsic errors and errors caused by ferromagnetic materials in the ambient.

EMT-based methods use landmarks or centerline to find a rigid transformation between EMT coordinate system and CT coordinate system. Using landmarks or centerline increases the overall bronchoscopy procedure time, which may be harmless for the patients. Furthermore, because of intrinsic EMT system errors and respiratory motion, these methods cannot track the bronchoscope accurately.

In 2000, Solomon et al. proposed and compared two EMT-based image registration methods for CT-guided bronchoscopy. These methods use real skin markers or the inner surface of the trachea for registration between EMT and CT space. They achieved $57 \%$ for total percentage of successfully registered frames (12).

Hybrid methods try to overcome the problems of traditional methods by combining them. In fact, they are supposed to achieve high speed of EMT-based methods and accuracy of image-based methods. These methods require landmark-based or centerline-based registration before main bronchoscopy which could be time consuming and hence unfavorable. After achieving a rigid transformation between CT and EMT coordinate system, VB image is searched in a smaller search space that best matches real bronchoscopic video frame.

In 2005, Mori et al. proposed a hybrid method for bronchoscope tracking. They used the EMT sensor position as the starting point for intensity based registration between real and virtual bronchoscopy images. The method was tested using a bronchial phantom model with simulated respiratory motion and a $64.5 \%$ percentage of total successfully registered frames was achieved (13).

In 2010, Luo et al. proposed a new scheme for hybrid bronchoscope tracking, and evaluated it on a dynamic motion phantom. Hybrid methods fail when the starting point acquired by EMT is too far from the actual pose. To overcome this problem, they used a threshold for Euclidean distance between current EM sensor position and the position acquired in CT. For this study, the percent- age of successfully registered frames was $75 \%$ (14). In another study in 2010, the same authors modified the above method by using a sequential Mont Carlo sampler to find an optimum starting point for intensity based registration, and the percentage of successfully registered frames was $92 \%$ (11).

In 2013, Holmes et al. proposed an image-based system for technician-free bronchoscopy guidance (15). They developed their work as a hands-free system in 2015 (16).

Hence our method uses real bronchoscopy images and EMT data simultaneously, it might be reported as a hybrid method that would overcome problems in other hybrid techniques. The proposed method matches the bronchoscopy image contours with the mapped CT contours at different positions to achieve the true position. Synchronous EMT data helps us to do this in less wrong positions, and achieve the true position faster.

\section{Objectives}

We aimed to develop a continuous guiding method for bronchoscopy with high tracking accuracy by matching bronchoscopy image contours with CT contours, and speed it up by using synchronous EMT data, and to evaluate it on airway phantom with simulated respiratory motion.

\section{Materials and Methods}

Real time bronchoscope tracking aims to find real camera position at CT space when each frame arrives.

As illustrated in Figure 1, CT data is modeled as contours in each branch. Then, a virtual camera pose is set using EMT pose relative to last frame's pose. Afterward, CT contour which is visible to the camera is mapped to virtual camera's image plane in a perspective way. If the contours that are detected in the current frame are matched to the mapped CT contours, registration is done and the system waits for the next frame. Otherwise, a new virtual camera pose is set, using the last camera pose of the current frame, and EMT pose is compared to the last frame.

\subsection{Data Acquisition}

Our setup for data acquisition consisted of Olympus bronchoscopy system, NDI Aurora EMT system, airway phantom with simulated respiratory motion, and a personal computer (PC).A BlackMagicDesign capture card was used to transfer video frames from bronchoscopy system to PC (Figure 2).

NDI Aurora EMT system has four components including electromagnetic (EM) sensors, EMT field generator, system control unit (SCU) which sends commands and sends 


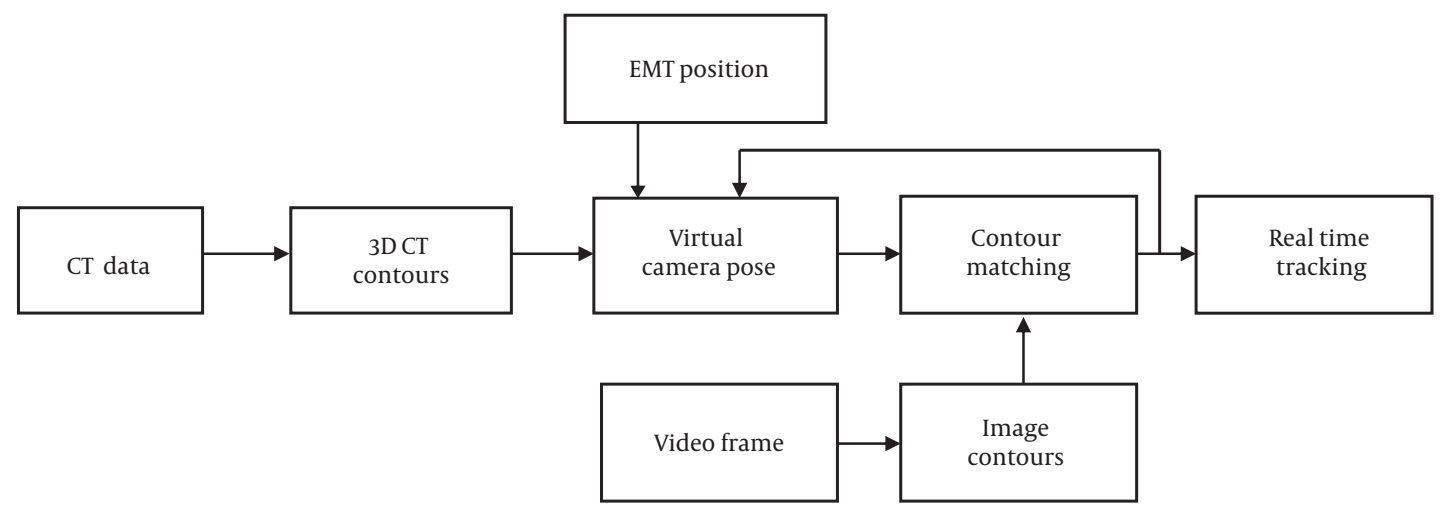

Figure 1. Block diagram of the proposed method (EMT,electromagnetic tracker).

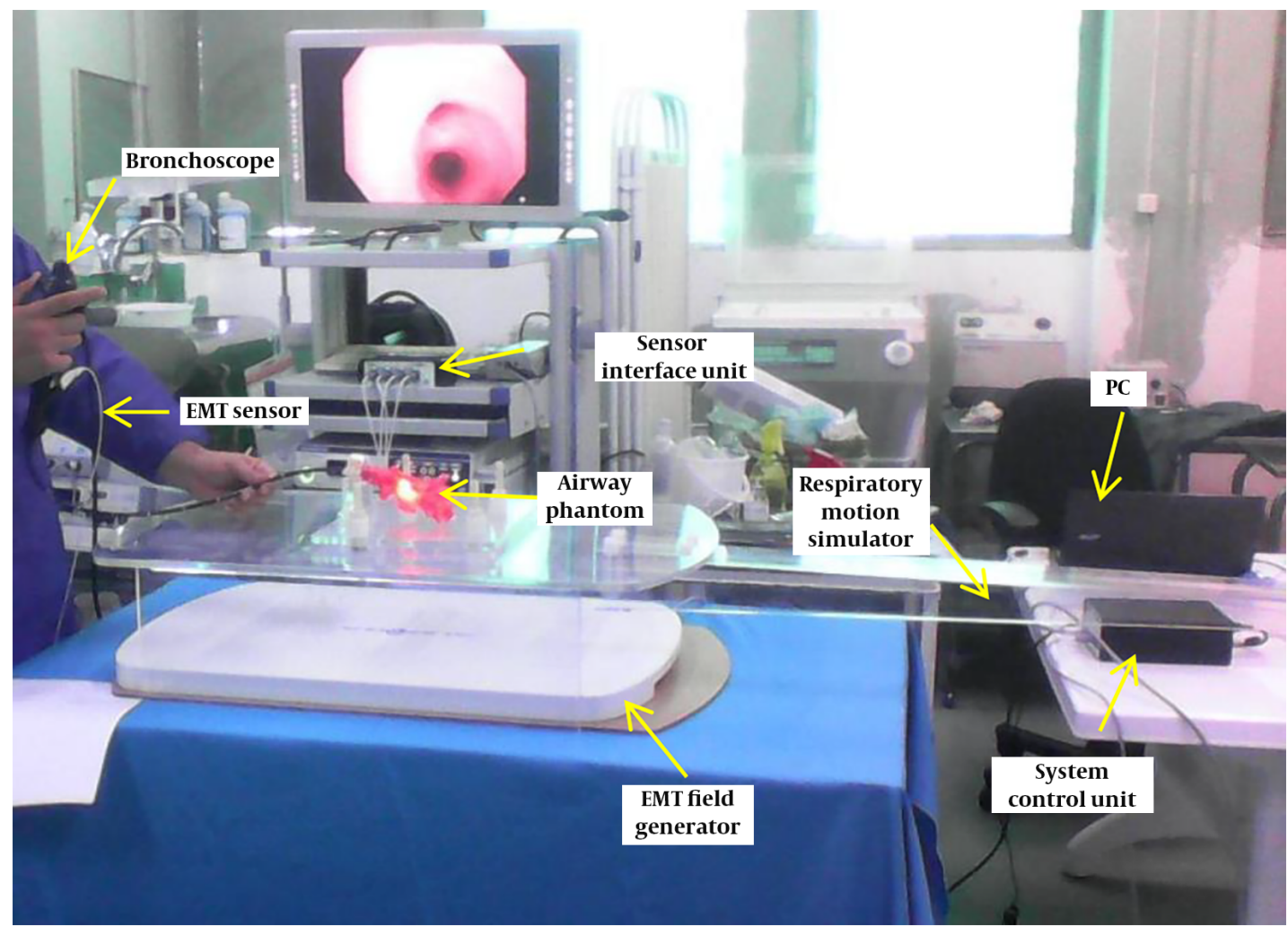

Figure 2. Data acquisition system consisted of bronchoscopy, electromagnetic tracker (EMT), airway phantom, respiratory motion simulator, and personal computer (PC) for recording

sensor information to PC, and sensor interface unit (SIU) that connects sensor(s) to SCU. The intrinsic error of sensor we used was less than $0.6 \mathrm{~mm}$. EM sensor was attached to the bronchoscope via the biopsy channel. The Olympus bronchoscopy system captures the video frames with a resolution of $576 \times 720$ pixels.
A software called SyVER (synchronized video-EMT recorder) was developed to synchronize EMT frames with live video frames from the capture card precisely. Frame rate was set to $15 \mathrm{~Hz}$. Each video frame was considered with its corresponding EMT frame (6 degree of freedom [DOF] position). 
A stepper motor was used to apply motion to the phantom and a $2 \mathrm{~mm} 6$ DOF EM sensor was fixed into the biopsy channel of bronchoscope so that its tip was at the tip of the bronchoscope.

At each trial, the bronchoscopist tried to maneuver the bronchoscope through all branches of the airway phantom. Twenty trials were performed by four experts with four different respiratory motions. Table 1 shows the trials.

\begin{tabular}{lccc}
\hline Table 1. Trials Performed for Sweeping Airway Tree with Bronchoscope \\
\hline $\begin{array}{l}\text { Maximal } \\
\text { motion }\end{array}$ & $\begin{array}{c}\text { Number of } \\
\text { trials }\end{array}$ & $\begin{array}{c}\text { Average trial } \\
\text { time }^{\mathbf{a}}(\mathbf{s})\end{array}$ & $\begin{array}{c}\text { Number of } \\
\text { frames }\end{array}$ \\
\hline $\mathbf{6} \mathbf{~ \mathbf { m }}$ & 5 & $127.9 \pm 7.8$ & 9591 \\
$\mathbf{1 2} \mathbf{~ \mathbf { ~ m }}$ & 5 & $134.6 \pm 12.1$ & 10095 \\
$\mathbf{1 8} \mathbf{~ \mathbf { m }}$ & 5 & $130.4 \pm 10.9$ & 9781 \\
\hline $\mathbf{2 4} \mathbf{~ m m}$ & 5 & $131.9 \pm 14.3$ & 9894 \\
\hline
\end{tabular}

${ }^{\mathrm{a}}$ Values are expressed as mean \pm standard deviation (SD).

Phantom CT data was acquired by a 16-slice Siemens CT scanner with pitch 1, $440 \mathrm{~mA}, 0.5 \mathrm{~mm}$ slice thickness and $0.5 \times 0.5 \mathrm{~mm}$ pixel size before the video-EMT data acquisition during live bronchoscopy.

\subsection{Contour Detection}

Shape analysis methods are widely used in object recognition, matching, registration, and analysis. A contour is defined as curve represented by a set of chained image points.

For processing of bronchoscopic video images, the image is first converted in gray because we used to work on grayscale picture. A simple threshold is applied to roughly get contours. The open and close trick is applied by a round 7-pixel structing element to get more smooth contours. Finally, a contour detection algorithm is applied as described by Suzuki, 1985 (17). Suzuki showed a way to analyze the topological structure of binary images by border following. The contour detection algorithm first determines border points, surroundness among connected components, outer border and hole border, and surroundness among borders. Then, it scans the input binary image with a raster and interrupts the raster when a border point is found following the starting point of either an outer border or a hole border, and assigns a uniquely identifiable number (NBD) to the newly found border. After following the entire border, the raster scan continues until it reaches the lower right corner of the image. The output is shown in Figure 3.

\subsection{CT Data Analysis}

CT data analysis can be performed offline, before real time tracking. 3D structure of airway tree phantom was segmented by thresholding CT data. Since extraction of the internal wall of the airway phantom is important, it was acquired manually slice by slice and then the whole structure of the internal wall was reconstructed. Afterward, the internal wall of the 3D structure of airway tree phantom should be modeled as contours. Subsequently, at each branch, two points were considered as starting point and endpoint of the branch. At each bifurcation, the endpoint of the main branch was considered as the starting point of bifurcated branches. Centerline of each branch was roughly considered as a line that connects the starting point to the endpoint. The centerline of each branch was divided into $1 \mathrm{~mm}$ segments. At each segment a plane was considered perpendicular to the line, and intersection of the plane with the structure of the internal wall was modeled as a CT contour. Therefore, the whole structure was modeled by 96 CT contours. Each CT contour saves its shape and 6 degree of freedom (DOF) position (Figure 4).

\subsection{Contour Based 2D/3D Registration}

For the purpose of real time tracking, we set a virtual camera at a 6 DOF position that maps its visible CT contours on its virtual plane using perspective projection (Figure 5). Virtual camera's intrinsic parameters were set to intrinsic parameters of the real camera that was acquired by a checkerboard pattern. When each video frame arrives, we look for a 6 DOF position of virtual camera that best matches mapped CT contours with the contours of live video frame.

Some methods for contour matching have been developed (18-20). For this purpose, after finding the contours, the epipolar geometry of two images is recovered, the contours are matched, and the false match is found. For false matches, the epipolar geometry is recomputed, and contours are rematched.

To recover epipolar geometry, we use Hartley's improved 8-point algorithm to compute the fundamental matrix $F_{i j}$. The fundamental matrix $F_{i j}$ is a $3 \times 3$ matrix satisfying

$x_{j k}^{T} F_{i j} x_{i k}=0, k=1, \ldots, n$

for any match pair in the two images $i$ and $j$.

In order to find the corresponding point $c_{j}\left(s^{-}{ }_{k}\right)$, we computed

$c_{j}\left(\dot{s}_{k}\right)=\arg _{l=1, \ldots, n} \min \left\|c_{j}^{T}\left(s_{l}\right) F_{i j} c_{i}\left(s_{k}\right)\right\|$

Afterward, for the contours which are not matched, the epipolar geometry is recomputed. 

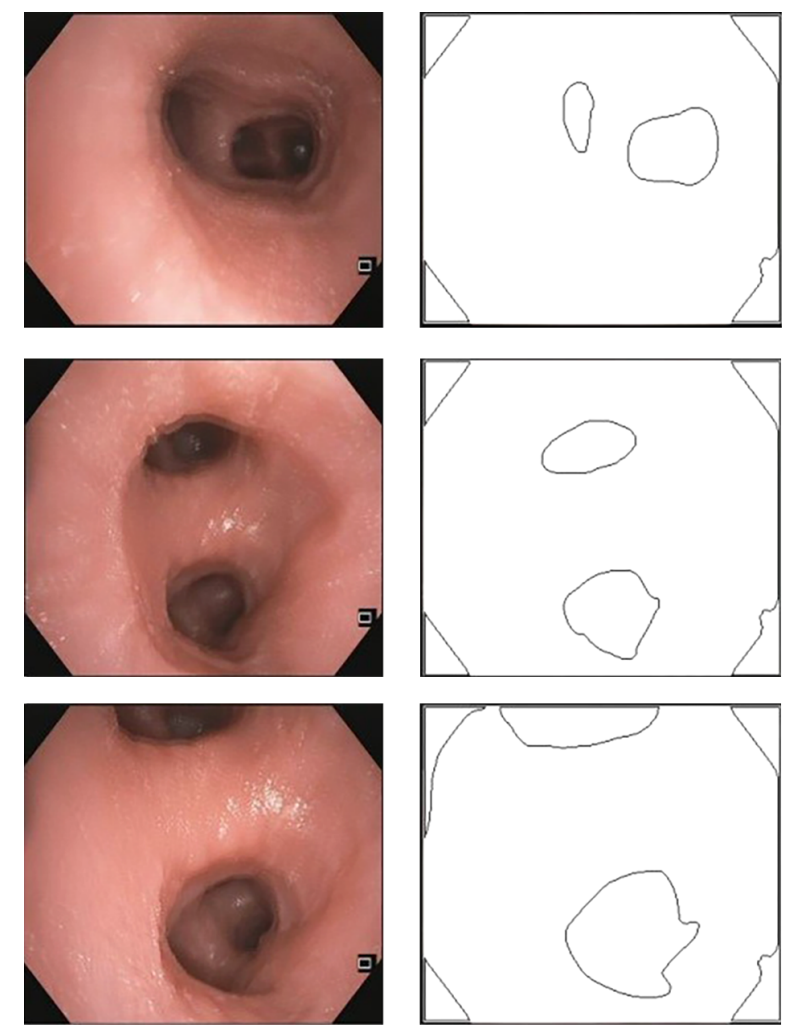
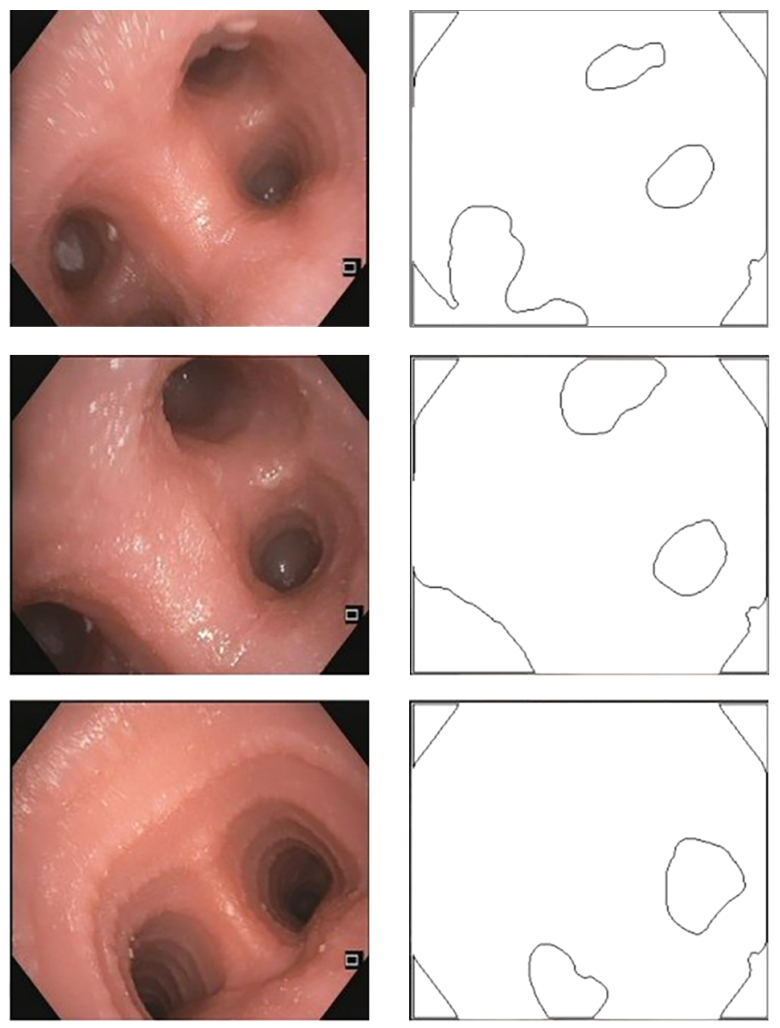

Figure 3. Real bronchoscopy image frames and results of contour detection

\subsection{Validation}

For validation of tracking accuracy, at each frame, virtual bronchoscopy (VB) image was generated at the position reported by the proposed method. Afterward, similarity between VB image and real video frame was measured using mutual information (MI) as follows (21):

$M I(V B, R B)=H(V B)+H(R B)-H(V B, R B)$

Where $H(A)$ represents the entropy of image $A$, and $M I(V B, R B)$ is the mutual information between VB image and real bronchoscopy $(\mathrm{RB})$ image. When the mutual information between $\mathrm{RB}$ and $\mathrm{VB}$ images is more than 0.73 of the entropy of RB image, we consider that they are similar images, and thus the image registration for that frame is successful. These criteria were set by an expert in a visual investigation scheme.

\section{Results}

CT data analysis was performed in MATLAB before real time data processing. Real time data analysis was performed on 20 trials with different respiratory motions using Microsoft visual studio C++. Each trial consisted of 1500
- 2100 frames, or 100 - $140 \mathrm{sec}$ at a $15 \mathrm{~Hz}$ frame rate. Each frame consists of a video frame and its synchronized EMT position.

Figure 6 shows bronchoscopy frames and CT-derived VBs generated at the position reported by the proposed method. For each trial, the accuracy was calculated as the ratio of successfully registered frames in the trial to number of frames of the trial. Finally, the overall accuracy was calculated as the ratio of successfully registered frames to total frames. Trial accuracy was considered to investigate respiratory motion effect on tracking accuracy. We compared our method with four other tracking schemes: 1) Solomon et al. (12) an EMT based method, 2) Mori et al. (13) a hybrid method, 3) Luo et al. (14) a hybrid method, and 4) Luo et al. (11) a hybrid method. Table 2 shows the percentage of successfully registered frames compared to others.

For investigation of the capability of real time tracking, we ran our algorithm for each trial on a PC with core i5 @1.8GHz and 4 GB RAM. This experiment shows that the total time needed to run the program and get the results is $91 \%$ of total live bronchoscopy time. So, this method can be used for real time tracking. 


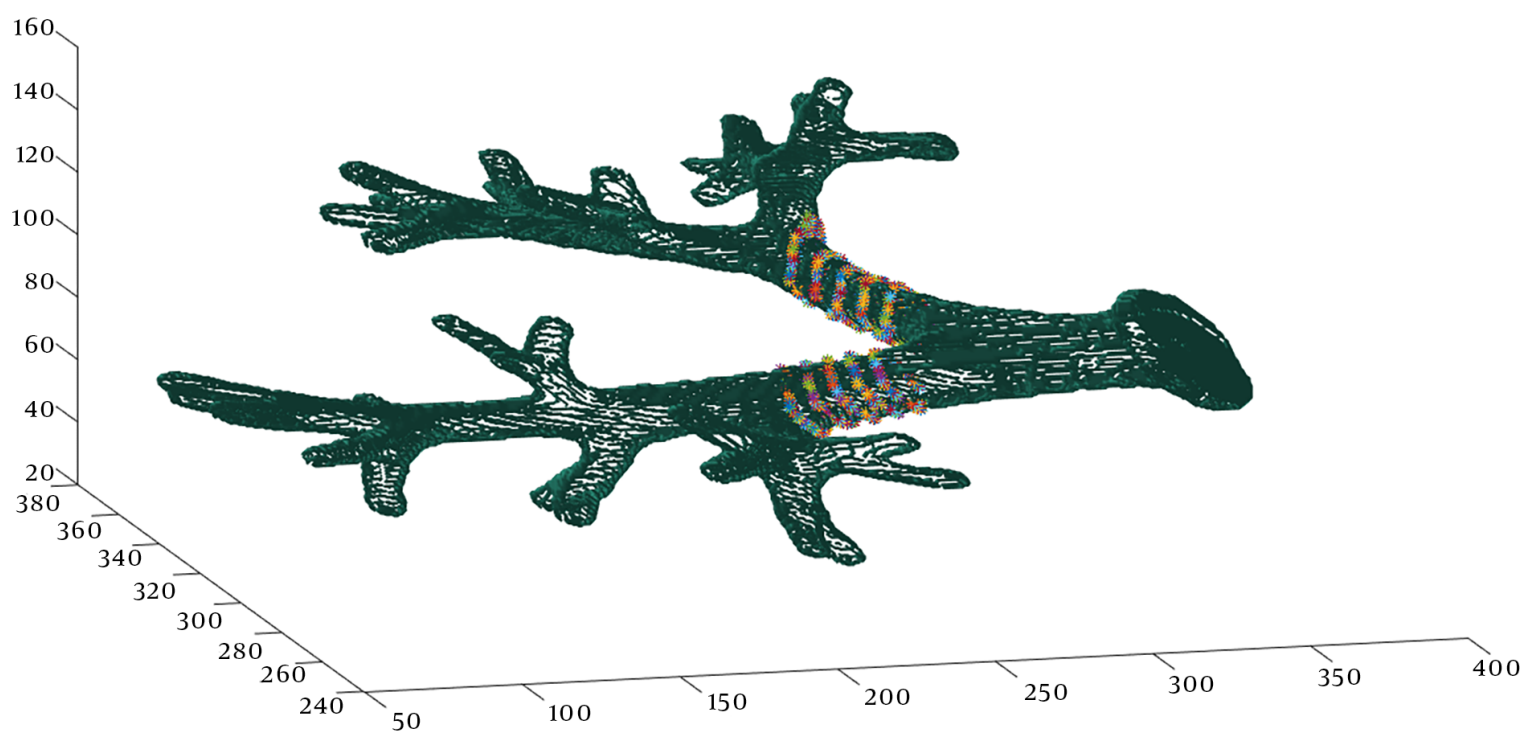

Figure 4. CT-derived airway tree. Some CT contours are shown in two branches. Scales are in $\mathrm{mm}$.

\begin{tabular}{|c|c|c|c|c|c|}
\hline \multirow{2}{*}{ Maximal motion } & \multicolumn{5}{|c|}{ Successfully registered frames, No. (\%) } \\
\hline & Solomon et al. (12) & Mori et al. (13) & Luo et al. (14) & Luo et al. (11) & Our method \\
\hline $6 \mathrm{~mm}$ & $850(66.1)$ & $958(74.6)$ & $1034(80.5)$ & $1224(95.3)$ & $9377(97.8)$ \\
\hline $12 \mathrm{~mm}$ & $783(59)$ & $863(65.1)$ & $1018(76.8)$ & $1244(93.8)$ & $9785(96.9)$ \\
\hline $18 \mathrm{~mm}$ & $894(56.8)$ & $972(61.8)$ & $1153(73.3)$ & $1431(91)$ & $9397(96.1)$ \\
\hline $24 \mathrm{~mm}$ & $716(48.8)$ & $850(57.9)$ & $1036(70.6)$ & $1300(88.6)$ & $9378(94.8)$ \\
\hline Total & $3243(57.4)$ & $3643(64.5)$ & $4241(75)$ & $5199(92)$ & 37919 (96.3) \\
\hline
\end{tabular}

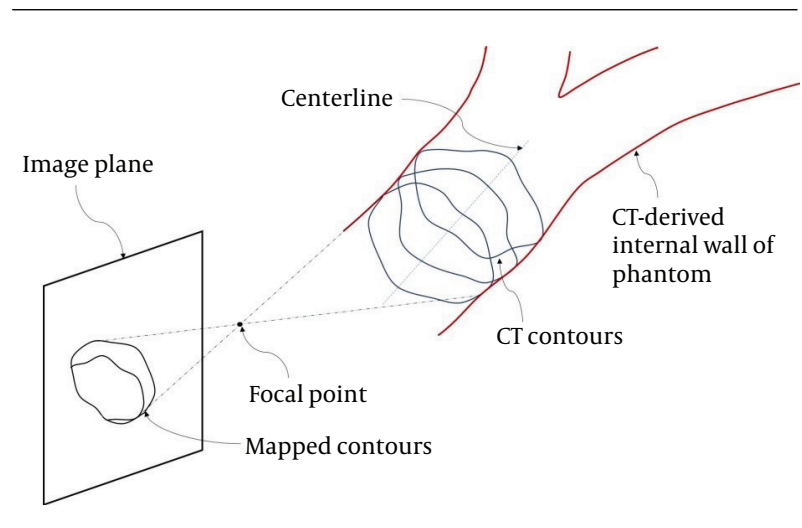

Figure 5. Schematic view of CT contours and their perspective projection on the image plane

\section{Discussion}

The aim of this study was to develop a hybrid bronchoscope tracking method that enables real time tracking with a high registration performance under respiratory motion, in particular, to deal with the limitations of imagebased registration speed. We used contour-based registration and EMT synchronous data. According to the results, the CT-video registration can be represented by contourbased registration with a high rate of successfully registered frames. The proposed method has a per frame registration speed of 1/15 of second, so the bronchoscope could be tracked real time by a rate of 15 frame/sec. Furthermore, the performance of frame registration is improved.

The previous method, landmark-based registration, is highly sensitive to respiratory motion. The proposed 

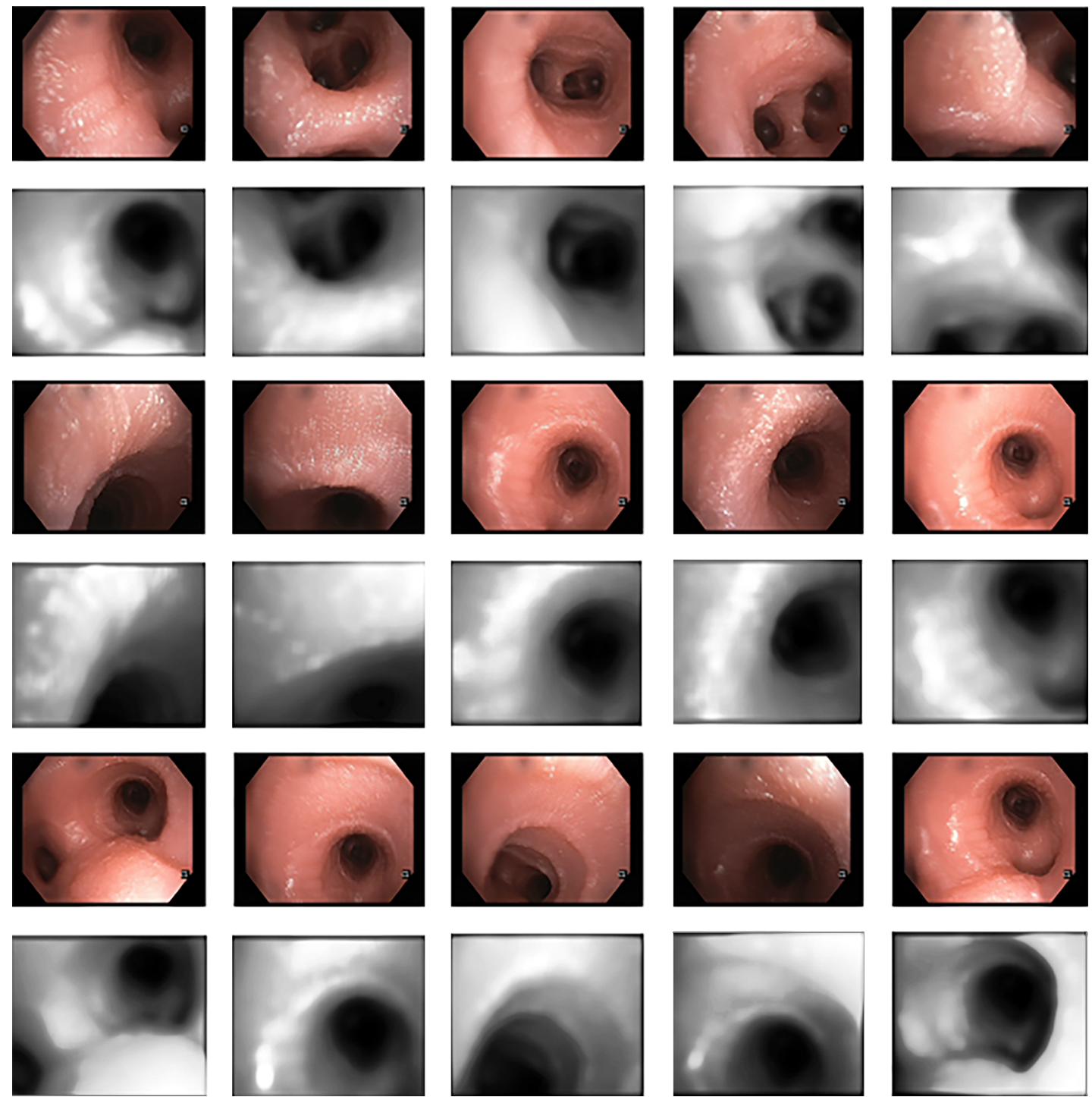

Figure 6. Real bronchoscopy image frames (odd rows), and CT derived virtual bronchoscopies (VB) generated at the position reported by this method (even rows)

method is robust to respiratory motion compared to other studies, as it uses EMT data in differential mode instead of landmark-based EMT data registration.

The initial pose of virtual camera from where image registration starts, may cause failure. Therefore, as for previous studies $(11,13,14)$, tracking accuracy suffers from dependencies on initialization of image registration. We have already suppressed it by using synchronous EMT data in differential mode. Since we use synchronous EMT data in differential mode, it may diverge when coughing or bubbling happens in the real airway structure. Additionally, bubbling may produce unwanted contours and cause failure in real conditions.
In conclusion, this paper presented a real time bronchoscope tracking method that used EMT data in differential mode, and contour matching, and evaluation on an airway tree phantom with simulated respiratory motion. The novelty of this work is developing a method that uses bronchoscopy images and EMT data in a unique way. Using contours, as useless information of bronchoscopy images, caused a faster registration procedure compared to intensity-based registration. Using differential approach for EMT data, it is not required to consider any landmark or centerline in the airway tree structure that is very difficult for the physician and consumes much more time for bronchoscopy. We strongly recommend using contour 
matching combined with differential EMT data instead of intensity-based image registration and landmark-based EMT registration. Respiratory motion errors are caused by point movement in a point-based registration approach. Because our method does not use point-based registration, it is respiratory motion error free.

In the future, we plan to perform an in vivo study to validate our method in real conditions to evaluate how much coughing and bubbling affect tracking accuracy.

\section{Footnotes}

Authors' Contributions: Study concept and design: Mehdi Samavati and Alireza Ahmadian; analysis and interpretation of data: Mehdi Samavati, Alireza Ahmadian, Amirhossein Golnabi, and Hamidreza Abtahi; drafting of the manuscript: Mehdi Samavati and Alireza Ahmadian; critical revision of the manuscript for important intellectual content: Mehdi Samavati, Alireza Ahmadian, Amirhossein Golnabi, and Hamidreza Abtahi; statistical analysis: Mehdi Samavati and Amirhossein Golnabi; hardware development and software design: Mehdi Samavati, Alireza Ahmadian, and Reza Arjmandi Asl

Conflict of Interests: It is not declared by the authors.

Ethical Considerations: The proposed method was evaluated on a phantom. Ethical consideration does not apply.

Financial Disclosure: The authors have no financial interests related to the material in the manuscript.

Funding/Support: This study was funded by Tehran University of Medical Sciences (TUMS) under grant No.: 9202-30-23128, and was supported by the Research Center for Biomedical Technologies and Robotics (RCBTR), Tehran, Iran.

\section{References}

1. Merritt SA, Khare R, Bascom R, Higgins WE. Interactive CT-video registration for the continuous guidance of bronchoscopy. IEEE Trans Med Imaging. 2013;32(8):1376-96. doi: 10.1109/TMI.2013.2252361. [PubMed: 23508260]. [PubMed Central: PMC3911781].

2. Miga MI, Sugiura T, Wong KH, Deguchi D, Feuerstein M, Kitasaka T, et al., editors. A method for accelerating bronchoscope tracking based on image registration by using GPU. Medical Imaging 2009: Visualization, Image-Guided Procedures, and Modeling. International Society for Optics and Photonics; 2009.

3. Luo X, Feuerstein M, Deguchi D, Kitasaka T, Takabatake H, Mori K Development and comparison of new hybrid motion tracking for bronchoscopic navigation. Med Image Anal. 2012;16(3):577-96. doi: 10.1016/j.media.2010.11.001. [PubMed: 21334250].

4. Bricault I, Ferretti G, Cinquin P. Registration of real and CT-derived virtual bronchoscopic images to assist transbronchial biopsy. IEEE Trans Med Imaging. 1998;17(5):703-14. doi:10.1109/42.736022. [PubMed: 9874294].

5. Deguchi D, Mori K, Feuerstein M, Kitasaka T, Maurer CR Jr, Suenaga Y, et al. Selective image similarity measure for bronchoscope tracking based on image registration. Med Image Anal. 2009;13(4):621-33. doi: 10.1016/j.media.2009.06.001. [PubMed:19592291].

6. Luo X, Wan Y, He X, Mori K. Adaptive marker-free registration using a multiple point strategy for real-time and robust endoscope electromagnetic navigation. Comput Methods Programs Biomed. 2015;118(2):147-57. doi: 10.1016/j.cmpb.2014.11.008. [PubMed: 25547498].

7. Feuerstein M, Sugiura T, Deguchi D, Reichl T, Kitasaka T, Mori K, editors. Marker-free registration for electromagnetic navigation bronchoscopy under respiratory motion. International Conference on Medical Imaging and Virtual Augmented Reality. Springer; 2010. p. 237-46.

8. Gergel I, dos Santos TR, Tetzlaff R, Maier-Hein L, Meinzer HP, Wegner I, editors. Particle filtering for respiratory motion compensation during navigated bronchoscopy. Medical Imaging 2010: Visualization, Image-Guided Procedures, and Modeling. International Society for Optics and Photonics; 2010.

9. Holmes Iii DR, Reichl T, Wong KH, Gergel I, Menzel M, Hautmann H, et al., editors. Real-time motion compensation for EM bronchoscope tracking with smooth output - ex-vivo validation. Proceedings of SPIE medical imaging. 2012.

10. Soper TD, Haynor DR, Glenny RW, Seibel EJ. In vivo validation of hybrid tracking system for navigation of an ultrathin bronchoscope within peripheral airways. IEEE Trans Biomed Eng. 2010;57(3):736-45. doi: 10.1109/TBME.2009.2034733. [PubMed: 19846362].

11. Luo X, Reichl T, Feuerstein M, Kitasak T, Mori K, editors. Modified hybrid bronchoscope tracking based on sequential monte carlo sampler: Dynamic phantom validation. Proceedings of the 10th Asian Conference on Computer Vision. Springer; 2010. p. 409-21.

12. Solomon SB, White P Jr, Wiener CM, Orens JB, Wang KP. Threedimensional CT-guided bronchoscopy with a real-time electromagnetic position sensor: A comparison of two image registration methods. Chest. 2000;118(6):1783-7. doi: 10.1378/chest.118.6.1783. [PubMed: 11115473].

13. Mori K, Deguchi D, Akiyama K, Kitasaka T, Maurer CR, Suenaga Y, et al., editors. Hybrid bronchoscope tracking using a magnetic tracking sensor and image registration. International Conference on Medical Image Computing and Computer-Assisted Intervention. Springer; 2005. $\mathrm{p}$ 543-50.

14. Luo X, Feuerstein M, Sugiura T, Kitasaka T, Imaizumi K, Hasegawa Y, et al., editors. Towards hybrid bronchoscope tracking under respiratory motion: Evaluation on a dynamic motion phantom. Proceedings of SPIE Medical Imaging. International Society for Optics and Photonics; 2010.

15. Holmes DR, Khare R, Yaniv ZR, Bascom R, Higgins WE, editors. Technician-free system for image-guided bronchoscopy. Proceedings of SPIE medical imaging; Image-Guided Procedures, Robotic Interventions, and Modeling. International Society for Optics and Photonics; 2013.

16. Khare R, Bascom R, Higgins WE. Hands-free system for bronchoscopy planning and guidance. IEEE Trans Biomed Eng. 2015;62(12):2794-811. doi: 10.1109/TBME.2015.2401514. [PubMed: 25675452]. [PubMed Central: PMC5560505].

17. Suzuki S. Topological structural analysis of digitized binary images by border following. Comput Vis Graph Image Process. 1985;30(1):32-46. doi: 10.1016/0734-189x(85)90016-7.

18. Park JS, Han JH. Contour matching: a curvature-based approach. Image Vis Comput. 1998;16(3):181-9. doi: 10.1016/s0262-8856(97)00064-4.

19. Adamek T, O'Connor N, editors. Efficient contour-based shape representation and matching. Proceedings of the 5th ACM SIGMM International Workshop On Multimedia Information Retrieval. ACM; 2003.

20. Han JH, Park JS. Contour matching using epipolar geometry. IEEE Trans Pattern Anal Mach Intell. 2000;22(4):358-70. doi: $10.1109 / 34.845378$.

21. Russakoff DB, Tomasi C, Rohlfing T, Maurer CR, editors. Image similarity using mutual information of regions. European Conference on ComputerVision. Springer; 2004. p. 596-607. 